\title{
Surface plasmon enhanced silicon solar cells
}

\author{
S. Pillai, ${ }^{a}{ }^{2}$ K. R. Catchpole, T. Trupke, and M. A. Green \\ ARC Photovoltaics Centre of Excellence, University of New South Wales, Sydney, Australia 2052
}

(Received 16 November 2006; accepted 20 March 2007; published online 7 May 2007)

\begin{abstract}
Thin-film solar cells have the potential to significantly decrease the cost of photovoltaics. Light trapping is particularly critical in such thin-film crystalline silicon solar cells in order to increase light absorption and hence cell efficiency. In this article we investigate the suitability of localized surface plasmons on silver nanoparticles for enhancing the absorbance of silicon solar cells. We find that surface plasmons can increase the spectral response of thin-film cells over almost the entire solar spectrum. At wavelengths close to the band gap of Si we observe a significant enhancement of the absorption for both thin-film and wafer-based structures. We report a sevenfold enhancement for wafer-based cells at $\lambda=1200 \mathrm{~nm}$ and up to 16 -fold enhancement at $\lambda=1050 \mathrm{~nm}$ for $1.25 \mu \mathrm{m}$ thin silicon-on-insulator (SOI) cells, and compare the results with a theoretical dipole-waveguide model. We also report a close to 12-fold enhancement in the electroluminescence from ultrathin SOI light-emitting diodes and investigate the effect of varying the particle size on that enhancement. (C) 2007 American Institute of Physics. [DOI: 10.1063/1.2734885]
\end{abstract}

\section{INTRODUCTION}

Silicon is the material of choice for photovoltaic applications due to its low cost, abundance in nature, nontoxicity, long-term stability, and well-established technology. The photovoltaic industry has seen extraordinary annual growth rates of over $30 \%$ in the last few years following improvements in the efficiency and the demand for alternative energy resources. However, for large-scale implementation, the cost of photovoltaic modules still has to be reduced significantly. One proposed approach toward such cost reduction is the use of very thin silicon absorbers in so-called thin-film or second-generation photovoltaic cells ${ }^{1-3}$ (the terminology first-generation photovoltaics is used for the more traditional wafer-based technology). For thin-film silicon solar cells, the $\mathrm{Si}$ absorber has a thickness on the order of only a few micrometers and is deposited on foreign substrates such as glass, ceramics, plastic, or metal for mechanical support. However, the efficiencies of such silicon thin-film cells at the moment are low compared to wafer-based silicon cells because of the relatively poor light absorption, as well as high bulk and surface recombination.

Because thin-film solar cells are only a few microns thick, standard methods of increasing the light absorption, which use surface textures that are typically around $10 \mathrm{mi}-$ crons in size, cannot be used. Plasma etch techniques, which can be used to etch submicron-sized features, can damage the silicon, thereby reducing the cell efficiency. Another alternative to direct texturing of $\mathrm{Si}$ is the texturing of the substrate. ${ }^{4}$ However, this also results in increased recombination losses through increased surface area. Though in practice it has been experimentally proven to be very difficult to reduce recombination losses beyond a certain limit, theoretically energy conversion efficiency of above $24 \%$ even for $1 \mu \mathrm{m}$ cells can be achieved. ${ }^{5}$ This highlights the need to incorporate

${ }^{a)}$ Electronic mail: supriyap@ @student.unsw.edu.au better light-trapping mechanisms that do not increase recombination losses in thin-film solar cells to extract the full potential of the cells.

In this article we demonstrate absorption and emission enhancement from Si solar cells by making use of the nanoscale properties of metals. This is an extension of our previous work on enhanced emission from $95 \mathrm{~nm}$ thick silicon-oninsulator (SOI) light-emitting diodes, which yielded an enhancement factor of 8 at a wavelength of $900 \mathrm{~nm} .{ }^{6}$ There has been little experimental work reported to date using surface plasmons in solar cell applications. Previous work with thick wafer-based solar cells has shown some enhancement in the visible wavelengths around $500-700 \mathrm{~nm}^{7}$ In this work we target enhancement close to the band gap of Si where absorption is weak, building on earlier work of Stuart et al., ${ }^{8}$ who reported an 18-fold enhancement at $800 \mathrm{~nm}$ for $160 \mathrm{~nm}$ thick SOI photodetectors. We investigate the effect of scattering by silver metal nanoparticles into $\mathrm{Si}$ devices and compare the absorption enhancement for thin silicon devices and thick wafer-based cells. Benefits of this light-trapping approach are clearly that the surface area of silicon and surface passivation layer remain the same as for a planar cell, so surface recombination losses are not expected to increase.

\section{BACKGROUND AND THEORY}

The electromagnetic properties of metal particles have been known for a long time since the work of Wood ${ }^{9}$ and Ritchie, ${ }^{10}$ but there has been renewed interest in recent years following the development of new nanofabrication techniques which makes it easy to fabricate these nanostructures. Localized surface plasmons (SP) are collective oscillations of the conduction electrons in metal particles. Movement of the conduction electrons upon excitation with incident light leads to a buildup of polarization charges on the particle surface. This acts as a restoring force, allowing a resonance to occur at a particular frequency, which is termed the dipole surface plasmon resonance frequency. 
Incident light that is in the region of the resonance wavelength of the particles is strongly scattered or absorbed, depending on the size of the particles. The extinction of the particles is defined as the sum of the scattering and absorption. For small particles in the quasistatic limit, the scattering and absorption cross sections are given by ${ }^{11}$

$$
C_{\text {abs }}=\frac{2 \pi}{\lambda} \operatorname{Im}[\alpha]
$$

and

$$
C_{\mathrm{sca}}=\frac{1}{6 \pi}\left(\frac{2 \pi}{\lambda}\right)^{4}|\alpha|^{2} .
$$

Here, $\alpha$ is the polarizability of the particle, given by $\alpha$ $=3 V(\varepsilon-1) /(\varepsilon+2)$ for a small spherical particle in vacuum, where $V$ is the volume of the particle and $\varepsilon$ is the permittivity of the metal. The scattering efficiency $Q_{\text {sca }}$ is given by $Q_{\text {sca }}$ $=C_{\text {sca }} /\left(C_{\text {sca }}+C_{\text {abs }}\right)$. Near the surface plasmon resonance, light may interact with the particle over a cross-sectional area larger than the geometric cross section of the particle because the polarizability of the particle becomes very high in this frequency range, as discussed in more detail elsewhere. ${ }^{12}$ Metals exhibit this property due to excitations of surface plasmons at the frequency where $\varepsilon \cong-2$. $^{11,12}$

Scattering and absorption depend on the size of the particles. Metallic particles that are much smaller than the wavelength of light tend to absorb more and hence extinction is dominated by absorption in the metal particles. Absorption dissipates heat and this property is utilized in applications like solar glazing, nanoscale lithography, and therapeutic applications. ${ }^{13,14}$ However, as the size of the particles increases, extinction is dominated by scattering and we take advantage of this property for our application of light trapping. Beyond certain limits, however, increasing the particle size leads to increased retardation effects and higher order multipole excitation modes, which decreases the efficiency of the scattering process.

A whole range of possibilities exist for tuning of surface plasmons. The resonance frequency of metal nanoparticles depends on the size, shape, particle material, and refractive index of the surrounding medium. ${ }^{15}$ In practice, larger size particles deposited on a substrate tend to lose their spherical property and hence look more like ellipsoids, causing the resonance frequency to be redshifted because of the change in shape. The resonance frequency also gets redshifted when the dielectric function of the surrounding medium increases. For example, the bulk plasmon resonance in vacuum changes when the same metal particles are deposited on a substrate. ${ }^{16}$ Furthermore, overcoating of the metal particles on a substrate can change the resonance. ${ }^{17}$ The particle material also affects the resonance frequency. For example, for a particle size corresponding to the same deposited mass thickness of gold and silver, gold nanoparticles have a lower frequency resonance than silver nanoparticles. Alloying the two metals in the different ratios can yield resonances in between the resonances of the pure gold and silver particles. ${ }^{18}$ The resonance can also be tuned using nanoshells that have a dielectric as the core and a metal as the shell. ${ }^{19}$ The redshifting of

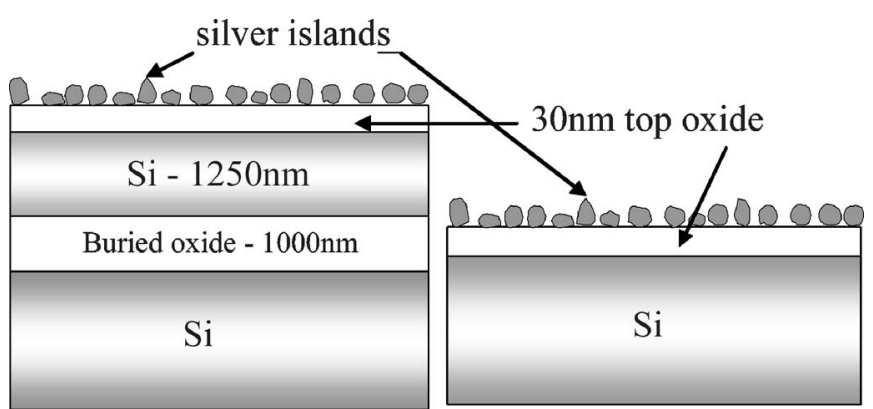

(a)

(b)

FIG. 1. The silicon cell structures used in the experiments. (a) Silicon-oninsulator with $1.25 \mu \mathrm{m}$ active $\mathrm{Si}$ and (b) wafer-based $300 \mu \mathrm{m}$ planar Si cell.

resonance leads to an increase in scattering cross section at longer wavelengths which would be beneficial for increasing the absorption of solar cells because of the indirect band gap of silicon. In this article we concentrate on the size/shape effects of the particles in tuning the resonance for our applications.

\section{METHOD}

Metal nanoparticles were deposited by thermal evaporation of thin layers of silver followed by annealing. This is one of the simplest methods of depositing metal particles onto a substrate. Silver is the metal of choice because of its low absorption losses when compared to other metals. The silver nanoparticles were formed by depositing varying mass thicknesses of a uniform layer of silver at the rate of approximately $2 \AA / s$ at $1 \times 10^{-5}$ Torr. The samples were then annealed in nitrogen at $200{ }^{\circ} \mathrm{C}$ for $50 \mathrm{~min}$. Due to surface tension, the particles coalesce together to form islands.

The samples used were (i) $1.25 \mu \mathrm{m}$ SOI test cells; (ii) planar PERL (passivated emitter rear locally diffused) $\mathrm{Si}$ cells (both as shown in Fig. 1); (iii) double-sided polished $\mathrm{Si}$ wafers; and (iv) plain glass. The SOI cells used in the experiments are formed from a lateral $p n$ junction diffused into a $p$-type layer. The $p$ and $n$ metal contacts were interdigitated for maximum carrier extraction with a cell dimension of 2 $\times 2 \mathrm{~cm}$. The thickness of the layers of the SOI devices as determined from reflectivity measurements and W-Vase simulation (software for spectroscopic ellipsometery data acquisition and analysis) were found to be $1248 \mathrm{~nm}$ for the silicon layer and $1000 \mathrm{~nm}$ for the buried oxide layer. The double-sided polished silicon sample was a $300 \mu \mathrm{m}$ thick wafer. The planar PERL cell was fabricated from a $1 \Omega \mathrm{cm}$ $p$-type float zone wafer and had an $n$-type passivated emitter with metal fingers in the front and the contact to the $p$-type at the rear, which also played the role of back reflector. The cell structure is similar to the one in Ref. 20 but without the "inverted pyramid" textures. All the samples had an approximately $30 \mathrm{~nm}$ top oxide which acts as a passivating layer, the thickness of which strongly influences the coupling between the active silicon layer and the plasmons. Surface recombination is a significant contributor to the total recombination in both types of devices and hence the passivation of the 
silicon surface is necessary, but the thickness of the oxide is kept low so as to provide good surface passivation without compromising the coupling to the silicon.

Different mass thicknesses of silver were deposited onto the samples to investigate the effect of particle size on the enhancement. Optical measurements in a spectrophotometer, electrical measurements in the form of spectral response $(A / W)$ measurements and photoluminescence (PL) imaging were used to characterize the samples. The optical measurements involved measuring the absorption from reflection and transmission measurements. The electrical measurements measured the short-circuit current from the sample per unit watt of incident light, as a function of wavelength. Optical measurements have the advantages that they are simple measurements and do not require finished devices, but they contribute to understanding of the process leading to the absorption enhancement. The optical and electrical measurements complement each other throughout the region of interest except close to the UV region where metals tend to absorb well. In this region we see absorption enhancement in the optical measurements (due to the absorption in the metal particles), but no corresponding increase in the electrical measurements (since there is no additional generation of electron-hole pairs in the silicon). Optical measurements on double-sided polished silicon wafers with layer dimensions similar to the planar PERL cell structures were used to compare with the results from electrical measurements of the planar PERL cell. Plain glass samples were used to find the bare island resonance of the nanoparticles for a particular deposition.

In the theoretical model, a point dipole approach was used for modeling the effect of the plasmons. ${ }^{21}$ The islands were treated as ideal, noninteracting dipoles emitting into the SOI waveguide. The model calculates the effect of the waveguide or substrate on both the angularly dependent emission spectrum and the angularly dependent scattering cross section, thereby developing a consistent point dipole view of the effect on absorbance and scattering. Experimental bare island resonance data were used for the initial scattering cross section for light incident from air, so that the large range of particle shapes and sizes (which broaden the surface plasmon resonance peak considerably) are taken into account. This was done because simple models of the extinction of metal islands only qualitatively reproduce the broadening seen experimentally. $^{22}$ The bare island data which are derived from metal islands on glass that were deposited simultaneously with the Si samples provide the closest easily obtainable approximation of the surface plasmon resonance for the range of shapes and sizes of the particles that would form on a silicon surface. The particles on the silicon samples can be assumed to have a similar dielectric environment to the particles on glass because there is a $\mathrm{SiO}_{2}$ layer of approximately $30 \mathrm{~nm}$ thickness on all the silicon samples, and the results of Bijeon et al. show that for this thickness of $\mathrm{SiO}_{2}$, the shift in the surface plasmon resonance due to an underlying silicon film is negligible. ${ }^{23}$ The model neglects absorption within the particles and all extinction is assumed to be scattering. Larger particles have a larger scattering cross section and, for our case where the particle sizes are greater than $100 \mathrm{~nm}$,

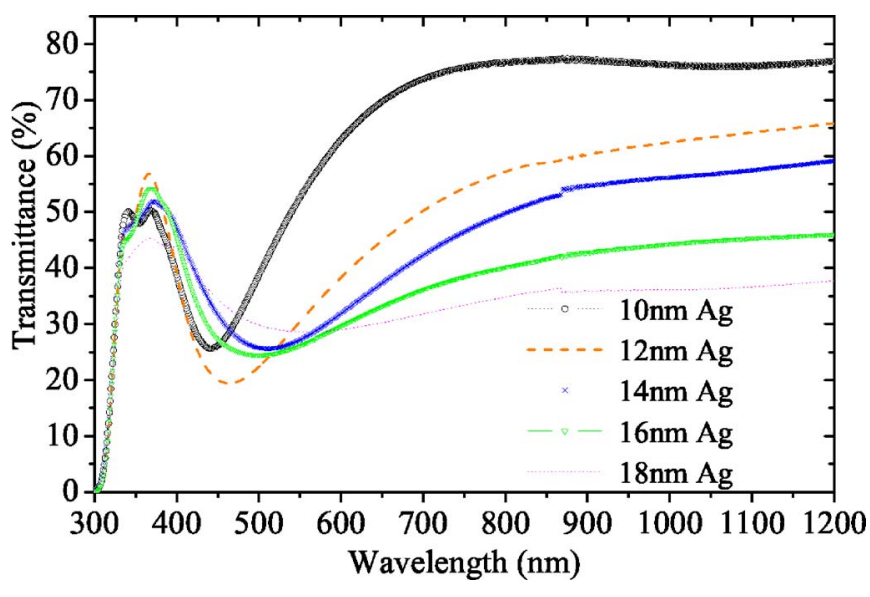

FIG. 2. Transmittance measurements showing redshifting of the bare island resonance of particles corresponding to increasing mass thickness of silver, which implies larger particle sizes.

absorption within the particles above wavelengths of $500 \mathrm{~nm}$ would be negligible as the radiative efficiency of these particles are high at longer wavelengths. ${ }^{8,21}$ A technique based on transfer matrices was used for tracing the flux coupled into the waveguide to calculate what fraction of that flux was absorbed, and what fraction was subsequently out-coupled by other metal islands. The overall effect was to treat the interaction of the metal particle with itself coherently, but the interaction with other metal particles incoherently.

\section{RESULTS}

The bare island resonance of the particles is the resonance of the particles on a low refractive index medium, so that interaction between the substrate and nanoparticles is minimized. We measure the bare island resonance of the silver nanoparticles on a plain microscope glass slide as the substrate. The particles formed as a result of annealing tend to become larger, more elongated, and also more irregular as the mass thickness of silver is increased. This causes the bare island resonance to shift to the red, as can be seen from the transmittance plots shown in Fig. 2. A dip in the transmittance plots shows the resonance position. The resonance curves are sharper for lower mass thicknesses and tend to broaden for larger mass thicknesses. This is likely to be due to averaging over the range of shapes of the nanoparticles and depolarization effects for larger particles. The bare island resonance plots give the extinction (1-transmittance) of the nanoparticles and are used as the input for the model to calculate the absorption enhancement for the structures used in the experiments.

Figure 3(a) shows the photocurrent enhancement from the 1.25 micron SOI test cells, which is defined as the ratio of the photocurrent response of the device measured after and before deposition of the silver islands. The interesting feature here is an overall increase in current throughout the visible and near-IR and a close to 16-fold enhancement at around $1050 \mathrm{~nm}$ with particle sizes corresponding to $16 \mathrm{~nm}$ mass thickness of silver. These results correspond to a $33 \%$ increase of the total current of the device, when averaged over the AM1.5 global radiation for particle sizes corre- 

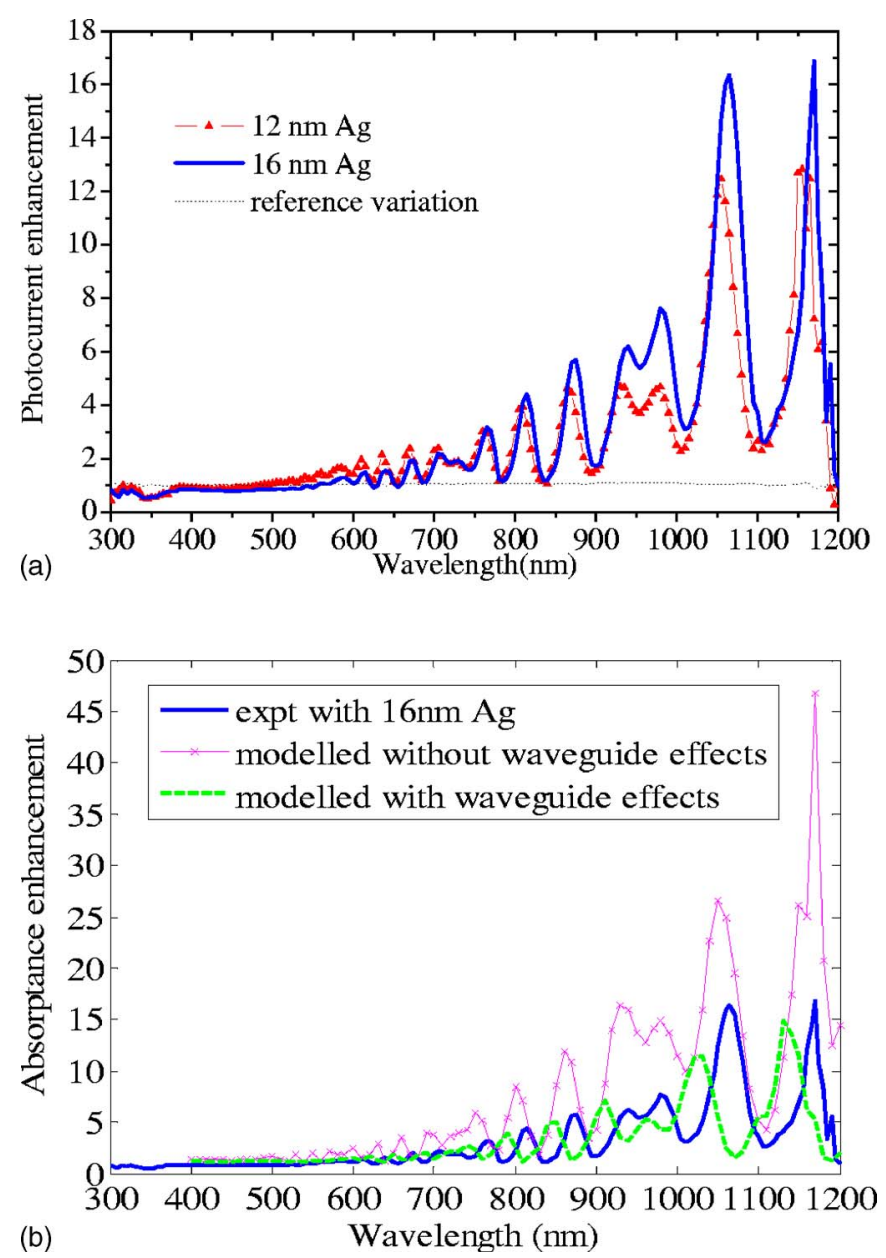

FIG. 3. (a) Photocurrent enhancement from a $1.25 \mu \mathrm{m}$ thick SOI test solar cell for particle sizes corresponding to 12 and $16 \mathrm{~nm}$ mass thickness of $\mathrm{Ag}$ relative to the cell without silver particles. (b) Photocurrent enhancement for the $1.25 \mu \mathrm{m}$ SOI device with $16 \mathrm{~nm}$ mass thickness silver particles, and modeled results with and without modification of the scattering cross section for normally incident light by the waveguide.

sponding to $12 \mathrm{~nm}$ mass thickness of silver and $16 \%$ increase for particle sizes corresponding to $16 \mathrm{~nm}$ silver thickness.

Figure 3(b) shows a comparison of the modeled and experimental results. The plot shows the effect of the plasmons both with and without the effect of the waveguide on the scattering cross section of the particles for externally incident light. (The effect of the waveguide on the scattering cross section for light incident from the waveguide modes is included in both cases of the modeled results, as this is a much stronger effect.) We can see that there is better agreement in the positions of the peaks when it is assumed that the waveguide does not modify the external scattering cross section. We have also found previously that this was the case when comparing experimental and modeled results for thinner SOI devices. ${ }^{21}$ The effect of including the waveguide modification for externally incident light is that the scattering cross section is enhanced at some wavelengths because the local field is enhanced due to constructive interference of the incident and reflected light, and decreased at other wavelengths due to destructive interference. The overall effect is a shift in the position of the enhancement peaks. The comparison of the experimental and modeled data suggests that, as was pre-
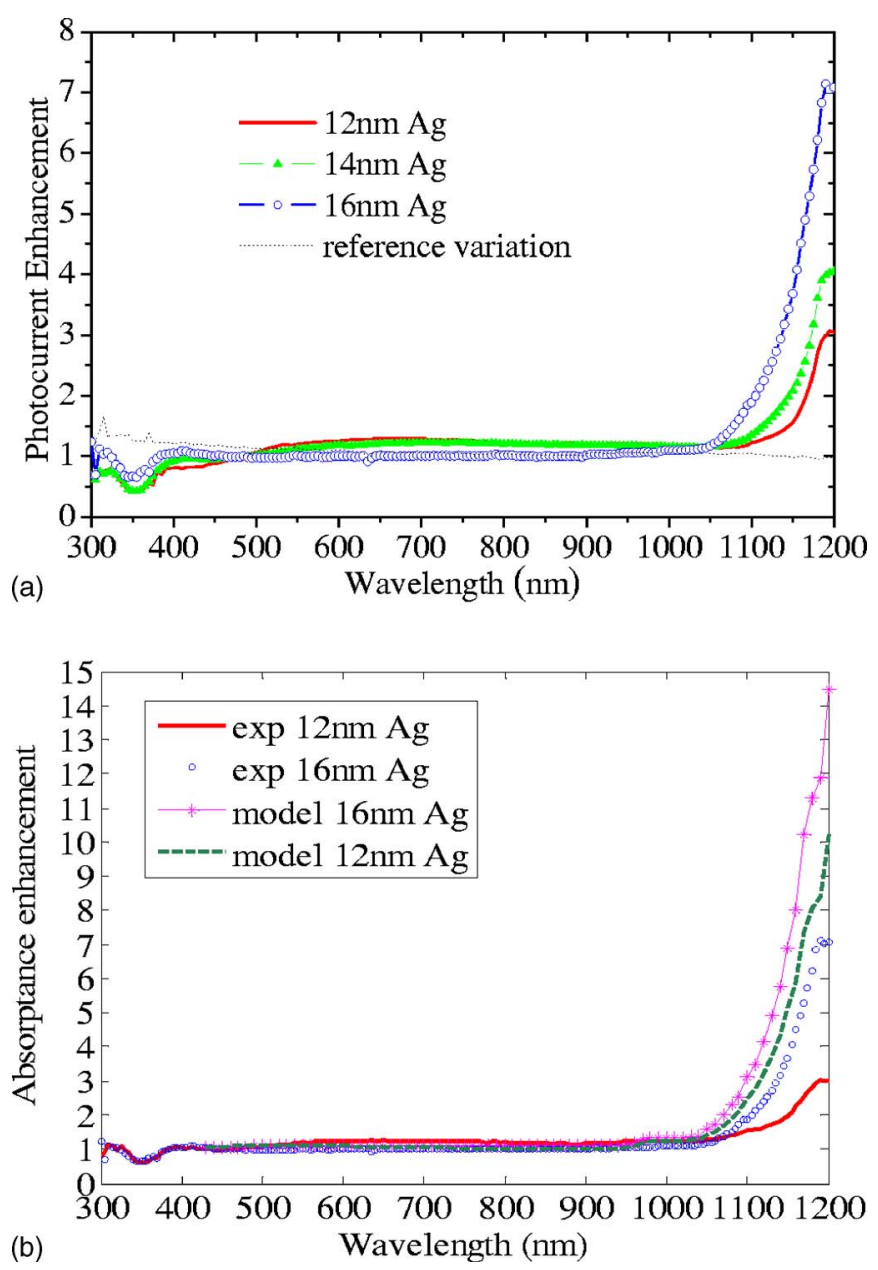

FIG. 4. (a) Photocurrent enhancement from a $300 \mu \mathrm{m}$ Si solar cell for particle sizes corresponding to 12,14 , and $16 \mathrm{~nm}$ mass thickness of $\mathrm{Ag}$ in comparison to the cell without silver particles. (b) Modeled enhancement result for a wafer-based planar silicon solar cell.

viously found, the modification of the external scattering cross section may be reduced for finite-sized particles compared to the modification for ideal dipoles. It is likely that the model predicts larger peaks than those observed experimentally because it is based on the bare island resonance data for particles on glass, which are known to be more elongated, with a larger variety of shapes (and hence which also have a broader surface plasmon resonance peak) than particles deposited on silicon with the equivalent deposition parameters.

Planar PERL cells were characterized before and after the deposition of silver nanoparticles in a process similar to the process described above on the SOI structures in order to study the effect of plasmons on thick wafer-based structures. The results are shown in Fig. 4(a). The corresponding increase in the photocurrent with the AM1.5 global spectrum for 12,14 , and $16 \mathrm{~nm}$ mass thickness of silver are approximately $19 \%, 14 \%$, and $2 \%$, respectively. Importantly, the photocurrent enhancement factor does not drop below unity in the entire spectral range $\lambda>500 \mathrm{~nm}$, which is important, because even a small relative reduction of the photocurrent over this broad spectral range would likely overcompensate the gains due to light trapping at wavelength near the band gap. The enhancement is more evident for larger-sized particles at longer wavelengths close to the band gap of $\mathrm{Si}$, 

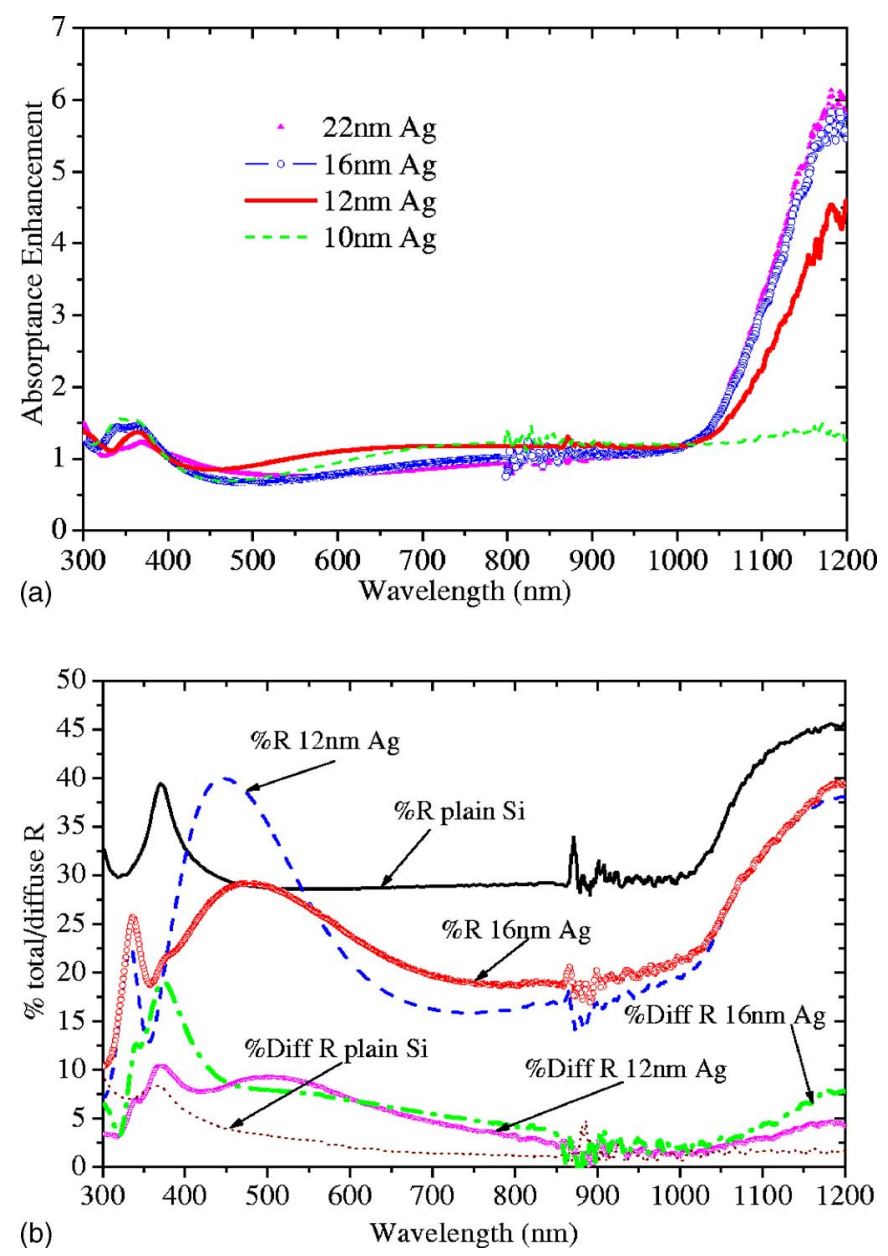

FIG. 5. (a) Enhancement from a double-sided polished Si wafer characterized optically using a spectrophotometer with particle sizes formed from different mass thickness of silver by thermal evaporation followed by annealing. (b) Total and diffuse reflectance plots from a double-sided polished Si wafer with $30 \mathrm{~nm}$ top oxide.

where the potential for enhancement is larger. However, relatively smaller-sized particles contribute to the enhancement in the entire wavelength range above $500 \mathrm{~nm}$, which explains the overall increase in enhancement for particles corresponding to $12 \mathrm{~nm}$ mass thickness of silver. The results show good agreement with the modeled result shown in Fig. 4(b). Here again, the larger enhancement in the model at long wavelengths seems likely to be due to a broader surface plasmon resonance for particles on glass than particles on Si. However, the fact that the experimental and modeled results agree well shows that the use of the unmodified scattering cross section as the input to the model is a good approximation.

Optical measurements on Si wafers with and without the islands showed the same trend as shown in Fig. 5(a). The optical measurements include absorption in metals as well, and the small peak at $350 \mathrm{~nm}$ is attributed to the region where metals tend to absorb quite strongly and reduce absorption in the silicon; this is shown as a dip in the photocurrent enhancement plot in Fig. 4(a).

\section{DISCUSSION}

While larger particles gave bigger enhancement at longer wavelengths, smaller particles gave increased absorption in the visible regime. From the results it can be seen that (a) enhancement is significant for thinner layers over the entire spectrum; and (b) with larger particle sizes there tends to be a reduction in absorption at shorter wavelengths, whereas at longer wavelengths there is an increase. However, for the wafer-based cells the enhancement in the visible region is not very prominent, whereas the enhancement is quite significant close to the band gap. One of the reasons for this is that the thicker wafer-based cells already absorb strongly in the visible and hence the effect of the plasmons is not significant. But, in contrast to the case for thin films, the impact of larger-sized particles for wafer-based cells is clearly evident. Smaller particles have a smaller scattering cross section and lower radiative efficiency, and hence tend to blueshift the absorption peak compared to larger particles with higher scattering cross section and radiative efficiency. However, there is an optimum size of the particles beyond which large particles tend to initiate multipole oscillations, which eventually tends to reduce the scattering efficiency. Hence, it is necessary to optimize the particle size to get an overall enhancement over a broad wavelength regime.

While thermal evaporation is an effective, well-controlled, and thus reproducible means of producing metal islands, ${ }^{24}$ we noted different results for our experiments for a given mass thickness when done in separate experimental runs. We attribute this to uncontrolled variations in the deposition and annealing conditions, and estimate the uncertainty due to the variation as being equivalent to a mass thickness variation of $2 \mathrm{~nm}$.

To further investigate the effect of increasing the size of the nanoparticles, silver of varying mass thickness was deposited on bare Si double-sided polished wafer and optically characterized by measuring the absorption in $\mathrm{Si}$ from the reflectance and transmittance data of the sample after annealing. It was evident from SEM images that the particle shape varied with increasing mass thickness. For thicker deposited metal layers the particles looked elongated and, as expected, the resonance showed a clear redshift, as was confirmed by the bare island resonance of these particles. It was also interesting to see the color variation of these islands, which could be easily distinguished by the naked eye. While the smallest particle sizes corresponding to $10 \mathrm{~nm}$ mass thickness of silver showed a deep blue color, the larger particles looked somewhat yellowish, indicating a clear shift in the reflectance peak (not shown here).

The absorption enhancement for the different sized particles can be seen in Fig. 5(a). The enhancement results from the 16 and $22 \mathrm{~nm}$ depositions were similar and large at long wavelengths, whereas for the $10 \mathrm{~nm}$ deposition the enhancement at long wavelengths was small. From Fig. 5(b) we can see that the particles act mostly as an antireflection coating in the visible regime, with reduced total reflectance in this wavelength range. At longer wavelengths the effect of light trapping dominates, with a stronger effect for larger particles. This is supported by the diffuse reflectance measurements shown in Fig. 5(b) for islands on wafers, as well as the extinction results for islands on glass (Fig. 2), which showed increased scattering from larger-sized particles when compared to smaller-sized particles. 


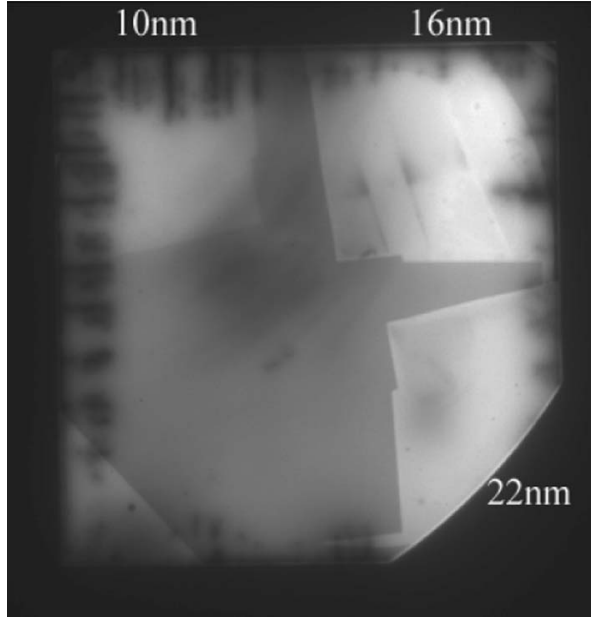

FIG. 6. Photoluminescence image for a $\mathrm{Si}$ wafer with islands corresponding to 10,16 , and $22 \mathrm{~nm}$ silver showing variation in the luminescence intensity. The intensity is lowest for the $10 \mathrm{~nm}$ case as the particle sizes are the smallest.

A photoluminescence imaging picture of the $\mathrm{Si}$ wafer is shown in Fig. 6. In this PL imaging a $815 \mathrm{~nm}$ laser is used to homogeneously illuminate the entire sample and excite $e-h$ pairs. The emitted PL signal is detected with a thermoelectrically cooled charge-coupled device (CCD) camera. $^{25}$ In Fig. 6 we can also see the much higher enhancement near the band gap of silicon for the 16 and $22 \mathrm{~nm}$ cases than the 10 $\mathrm{nm}$ case. The similarity of the enhancement in absorption for the 16 and $22 \mathrm{~nm}$ cases as seen in Fig. 5(a) is also evident in the similar intensity in the PL image.

The enhancement seen from the devices depends not only on the scattering efficiency of the particles but on waveguide or substrate effects as well. Waveguide effects include the number of waveguide modes the waveguide can support and on interaction of these modes with the plasmon modes, while substrate effects depend on the refractive index of the substrate. The incident light excites surface plasmon modes on the metal nanoparticles, which couple the light into the silicon, trapping most of the light inside until it is absorbed or coupled out by another metal nanoparticle. For both thin waveguides and thick devices, the dipole field around the metal nanoparticles contains high in-plane wave vector components that are evanescent in air but can propagate in silicon. If the metal nanoparticles are sufficiently close to the $\mathrm{Si}$, the evanescent field due to these components penetrates into the $\mathrm{Si}$ and becomes trapped, propagating modes. For nanoparticles on a thick silicon substrate, the high refractive index of the silicon means that most of the light scattered by the nanoparticles is radiated into the silicon (e.g., $87 \%$ at $\lambda$ $=900 \mathrm{~nm}$ for a horizontal electric dipole). For light well above the band gap energy of silicon, the absorption length within the silicon is smaller than the device thickness. The effect of the metal nanoparticles in this wavelength region is to reduce the reflectance of the device, due to the low fraction of light emitted into air, compared to the reflectance of bare silicon of around $30 \%$. However, close to the band gap of silicon, the absorption length within the silicon is larger than the device thickness. The extinction of the particles in this wavelength range is greater for larger particles, as can be seen from the bare island plots in Fig. 2 and from diffuse reflectance results as well. Because a large fraction of the light emitted into the silicon propagates as high in-plane wave vector, trapped modes, the absorption enhancement is increased in this wavelength region, as both the fraction of light coupled into the silicon, and the absorption of light that has been coupled in, are increased. For metal nanoparticles on a thin waveguide, some additional effects come into play. Reference 26 theoretically shows that the coupling between surface plasmon modes and waveguide modes is strongest at the modal cutoff frequencies. When the mode is just above cutoff, a large portion of the power carried by the mode is outside the Si layer and hence there is more interaction between the particle and the waveguide mode. This leads to the peaks seen in the photocurrent enhancement spectra for the SOI samples.

There is also the effect of the particle resonance which decides the absorption peak. From the bare island resonance plots in Fig. 2, it can be clearly seen that the scattering crosssection-which is proportional to the extinction-is redshifted as the particle size and shape vary. The total enhancement is proportional to the product of the waveguide contribution (light in-coupling and out-coupling, as well as effects of the waveguide on the scattering cross section) and the particle scattering effects (which depend on the size and shape of the individual particles). This explains the redshift of the enhancement peak at longer wavelengths for largersized particles clearly seen in Fig. 3.

For spherical particles the size of the particle does not significantly affect the resonance position, but affects just the scattering intensity. ${ }^{11}$ However, for our particles, the SEM images clearly show that as the mass thickness increases, the particles tend to lose their spherical shape and become flattened; hence, an increase in size of the particles for our case is accompanied by change in shape as well. The change in shape is due to the reduction in the coalescing forces as the mass thickness increases (for a fixed temperature in our case). The flattening of the particles and the range of shapes will tend to redshift and broaden the plasmon resonance, which we observe in the bare island resonance plots. The redshift is small for spherical particles but is larger when the particles get flatter, irrespective of the substrate. ${ }^{27}$ The 10 and $12 \mathrm{~nm}$ mass thicknesses had close to spherical shape particles for our case, and the proximity of the bare island resonance position for the two cases is in agreement with this observation.

As per the generalized form of Kirchhoff's law, which is valid for luminescent emission, enhanced emission corresponds to enhanced absorption. ${ }^{28}$ For emission, only a small fraction of the waveguided or internally generated light is coupled out of the waveguide in each interaction with the metal nanoparticles, but because the band-edge light is very weakly absorbed, after multiple scattering events the emission is significantly enhanced. This would imply that the results of enhanced absorption with larger-sized particles would be highly beneficial for applications like light emission. These results motivated us to try larger particles in conjunction with thin SOI LEDs. The LEDs have an active silicon layer thickness of $95 \mathrm{~nm}$ with a $408 \mathrm{~nm}$ thick buried 


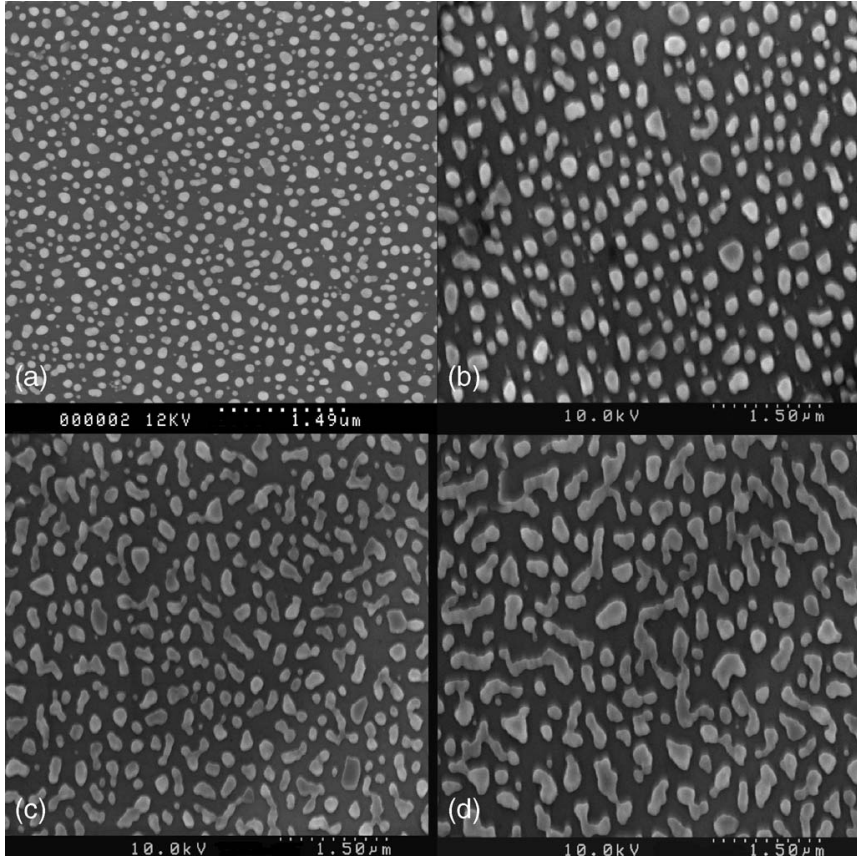

FIG. 7. SEM pictures showing silver metal particles corresponding to a mass thickness of (a) $14 \mathrm{~nm}$, (b) $16 \mathrm{~nm}$, (c) $18 \mathrm{~nm}$, and (d) $27 \mathrm{~nm}$ of silver.

oxide. Larger particle sizes corresponding to mass thicknesses of 16,18 , and $27 \mathrm{~nm}$ were deposited onto the SOI LEDs and the samples annealed in nitrogen for $50 \mathrm{~min}$. The variation in the size and shape of the particles with increasing mass thickness of silver can be seen in the SEM images in Fig. 7. Image analysis of these SEM pictures shows the particle sizes (major axis) to vary from $120 \mathrm{~nm}$ for the $14 \mathrm{~nm}$ case to $350 \mathrm{~nm}$ for the $27 \mathrm{~nm}$ case. The corresponding EL enhancement plots, with a clear redshift in the peak with increasing particle sizes, are shown in Fig. 8. For the case corresponding to the largest particle size, the overall enhancement integrated across the emission spectrum was 7 times, which is a significant improvement over our earlier results, where the enhancement for particle sizes corresponding to $14 \mathrm{~nm}$ mass thickness gave an overall enhancement of 3 times. $^{6}$

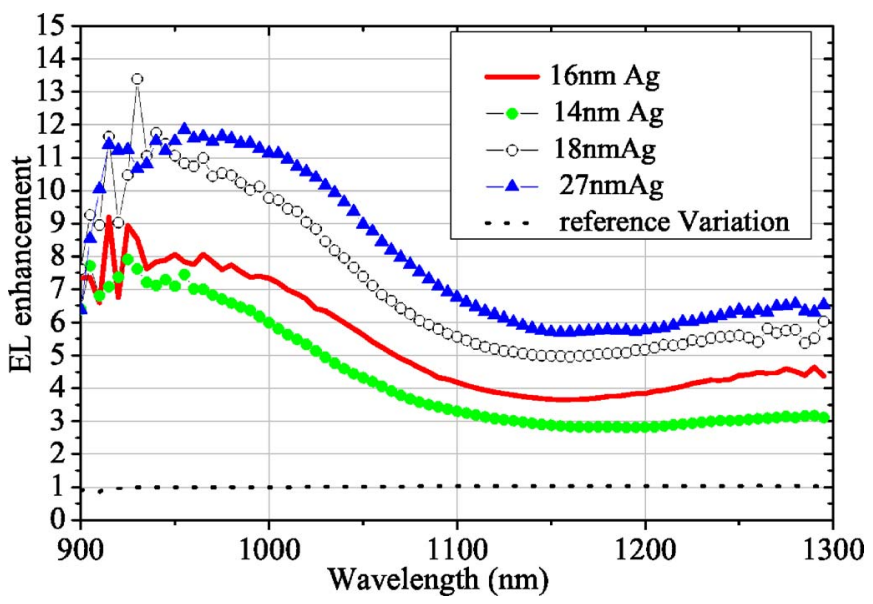

FIG. 8. EL enhancement plots for particle sizes corresponding to different mass thickness of silver showing an increased enhancement for larger particle sizes and a clear redshift in the enhancement peaks as the particle size increases.
The enhancement results for the thin film SOI lightemitting diodes with larger particles are very encouraging. Though the results with similar sized particles on waferbased cells do not look as promising except close to the band gap of silicon, the potential of the surface plasmons for thick wafer-based cells can be optimally utilized by depositing the particles on the rear of the cell. This would allow an antireflection coating to be deposited on the front to couple in shorter wavelength light, while surface plasmons on large metal nanoparticles at the rear of the cell could be utilized to trap light in the region close to the band gap of silicon. With this arrangement, any light in the blue which would possibly be absorbed in the metal if the nanoparticles were on the front side would instead be absorbed in the emitter, potentially increasing the absorption of the solar cell in the entire spectrum of interest. The same argument also holds for thinfilm cells.

The method described here is promising in terms of improving absorption and emission close to the band gap of silicon. Low absorption close to the band gap of silicon is a significant issue for solar cells due to the indirect band gap of $\mathrm{Si}$, and this approach can help boost the efficiency of Si cells. Plasmons could possibly be used with any semiconductor material where the band gap energy is less than the resonance frequency of the plasmons. A major advantage of the surface plasmon approach is that, unlike texturing of the silicon, the application of the metal nanoparticles will not degrade the electrical characteristics of the device, since they are separated from the silicon by a thin spacer layer. The metal islands are deposited by a simple process of evaporation and low-temperature annealing, and can be formed as the last stage of the device processing so there are no fundamental processing incompatibilities.

\section{CONCLUSION}

We have investigated the effect of surface plasmons on silver nanoparticles as a means of improving the efficiency of thin-film and wafer-based solar cells. Our results suggest that surface plasmons offer a promising way to improve the efficiency of thin-film solar cell structures, avoiding the problem of increased recombination which occurs when silicon is textured directly. This method also has the scope of further reducing the thickness of Si to below $1.5 \mu \mathrm{m}$ with good light trapping provided by the metal nanoparticles. Our results show that for front surface application, smaller metal particles provide maximum overall enhancement in the visible as well as the near-IR for solar cell applications, but that larger metal particles would be more beneficial for light emission from both thin and thick Si LEDs.

\section{ACKNOWLEDGMENTS}

The Photovoltaic Centre of Excellence is supported under the Australian Research Council's Centres of Excellence Scheme. S. Pillai would like to acknowledge the UNSW Faculty of Engineering Research Scholarship. K.R. Catchpole acknowledges the support of an Australian Research Council 
fellowship. The authors would like to thank Dr. J. Zhao for fabricating the SOI and planar PERL cells, and Daniel Inns for helping with the SEM images.

${ }^{1}$ M. A. Green, Sol. Energy 76, 3 (2004).

${ }^{2}$ M. A. Green, P. A. Basore, N. Chang, D. Clugston, R. Egan, R. Evans, D. Hogg, S. Jarnason, M. Keevers, P. Lasswell, J. O’Sullivan, U. Schubert, A. Turner, S. R. Wenham, and T. Young, Sol. Energy 77, 857 (2004).

${ }^{3}$ A. G. Aberle, J. Cryst. Growth 287, 386 (2006).

${ }^{4}$ M. J. McCann, K. R. Catchpole, K. J. Weber, and A. W. Blakers, Sol. Energy Mater. Sol. Cells 68, 135 (2001).

${ }^{5}$ M. A. Green, Prog. Photovoltaics 7, 327 (1999).

${ }^{6}$ S. Pillai, K. R. Catchpole, T. Trupke, G. Zhang, J. Zhao, and M. A. Green, Appl. Phys. Lett. 88, 161102 (2006).

${ }^{7}$ D. M. Schaadt, B. Feng, and E. T. Yu, Appl. Phys. Lett. 86, 063106 (2005).

${ }^{8}$ H. R. Stuart and D. G. Hall, Appl. Phys. Lett. 73, 3815 (1998).

${ }^{9}$ R. W. Wood, Proc. Phys. Soc. London 18, 166 (1902).

${ }^{10}$ R. H. Ritchie, Surf. Sci. 34, 1 (1973).

${ }^{11}$ C. F. Bohren and D. R. Huffman, Absorption and Scattering of Light by Small Particles (Wiley-Interscience, New York, 1983).

${ }^{12}$ C. F. Bohren, Am. J. Phys. 51, 323 (1983).

${ }^{13}$ M. Cortie, X. Xu, H. Chowdhury, H. Zareie, and G. Smith, Proc. SPIE 5649, 565 (2005).
${ }^{14}$ D. Pissuwan, S. Valenzuela, and M. B. Cortie, Trends Biotechnol. 24, 62 (2006).

${ }^{15}$ U. Kreibig and M. Vollmer, Optical Properties of Metal Clusters (Wiley, New York, 1995).

${ }^{16}$ P. Royer, J. P. Goudonnet, R. J. Warmack, and T. L. Ferrel, Phys. Rev. B 35, 3753 (1987).

${ }^{17}$ G. Xu, M. Tazawa, P. Jin, S. Nakao, and K. Yoshimura, Appl. Phys. Lett. 82, 3811 (2003).

${ }^{18}$ K. Baba, T. Okuno, and M. Miyagi, Appl. Phys. Lett. 62, 437 (1993).

${ }^{19}$ S. J. Oldenburg, R. D. Averitt, S. L. Westcott, and N. J. Halas, Chem. Phys. Lett. 288, 243 (1998).

${ }^{20}$ J. Zhao, A. Wang, and M. A. Green, Prog. Photovoltaics 7, 471 (1999).

${ }^{21}$ K. R. Catchpole and S. Pillai, J. Appl. Phys. 100, 044504 (2006).

${ }^{22}$ T. Gotz, W. Hoheisel, M. Vollmer, and F. Trager, Z. Phys. D: At., Mol. Clusters 33, 133 (1995).

${ }^{23}$ J. L. Bijeon, P. Royer, J. P. Goudonnet, R. J. Warmack, and T. L. Ferrell, Thin Solid Films 155, L1 (1987).

${ }^{24}$ R. Gupta, M. J. Dyer, and W. A. Weimer, J. Appl. Phys. 92, 5264 (2002).

${ }^{25}$ T. Trupke, R. A. Bardos, M. C. Schubert, and W. Warta, Appl. Phys. Lett. 89, 044107 (2006).

${ }^{26}$ B. J. Soller and D. G. Hall, J. Opt. Soc. Am. A 18, 2577 (2001).

${ }^{27}$ P. Royer, J. L. Bijeon, J. P. Goudonnet, T. Inagaki, and E. T. Arakawa, Surf. Sci. 217, 384 (1989).

${ }^{28}$ P. Wurfel, J. Phys. C 15, 3967 (1982). 